\title{
Colligative Properties of Solutions:
}

\section{Vanishing Concentrations}

\author{
Kenneth S. Alexander, ${ }^{1}$ Marek Biskup, ${ }^{2}$ and Lincoln Chayes $^{2}$
}

Received July 15, 2004; accepted December 2, 2004

\begin{abstract}
We continue our study of colligative properties of solutions initiated in ref. 1 . We focus on the situations where, in a system of linear size $L$, the concentration and the chemical potential scale like $c=\xi / L$ and $h=b / L$, respectively. We find that there exists a critical value $\xi_{\mathrm{t}}$ such that no phase separation occurs for $\xi \leqslant \xi_{\mathrm{t}}$ while, for $\xi>\xi_{\mathrm{t}}$, the two phases of the solvent coexist for an interval of values of $b$. Moreover, phase separation begins abruptly in the sense that a macroscopic fraction of the system suddenly freezes (or melts) forming a crystal (or droplet) of the complementary phase when $b$ reaches a critical value. For certain values of system parameters, under "frozen" boundary conditions, phase separation also ends abruptly in the sense that the equilibrium droplet grows continuously with increasing $b$ and then suddenly jumps in size to subsume the entire system. Our findings indicate that the onset of freezing-point depression is in fact a surface phenomenon.
\end{abstract}

KEY WORDS: Freezing-point depression; phase separation; droplet transition; Wulff construction; Ising model; canonical ensemble.

\section{INTRODUCTION}

\subsection{Overview}

In a previous paper (ref. 1, henceforth referred to as Part I) we defined a model of non-volatile solutions and studied its behavior under the conditions when the solvent undergoes a liquid-solid phase transition. A particular example of interest is the solution of salt in water at temperatures

\footnotetext{
${ }^{1}$ Department of Mathematics, USC, Los Angeles, California, USA.

${ }^{2}$ Department of Mathematics, UCLA, Los Angeles, California, USA, e-mail: biskup@ math.ucla.edu
} 
near the freezing point. In accord with Part I we will refer to the solute as salt and to the two phases of solvent as ice and liquid water.

After some reformulation the model is reduced to the Ising model coupled to an extra collection of variables representing the salt. The (formal) Hamiltonian is given by

$$
\beta \mathscr{H}=-J \sum_{\langle x, y\rangle} \sigma_{x} \sigma_{y}-h \sum_{x} \sigma_{x}+\kappa \sum_{x} S_{x} \frac{1-\sigma_{x}}{2} .
$$

Here we are confined to the sites of the hypercubic lattice $\mathbb{Z}^{d}$ with $d \geqslant 2$, the variable $\sigma_{x} \in\{+1,-1\}$ marks the presence of liquid water $\left(\sigma_{x}=1\right)$ and ice $\left(\sigma_{x}=-1\right)$ at site $x$, while $S_{x} \in\{0,1\}$ distinguishes whether salt is present $\left(S_{x}=1\right)$ or absent $\left(S_{x}=0\right)$ at $x$. The coupling between the $\sigma$ 's is ferromagnetic $(J>0)$, the coupling between the $\sigma$ 's and the $S$ 's favors salt in liquid water, i.e., $\kappa>0-$ this reflects the fact that there is an energetic penalty for salt inserted into the crystal structure of ice.

A statistical ensemble of direct physical - and mathematical - relevance is that with fluctuating magnetization (grand canonical spin variables) and a fixed amount of salt (canonical salt variables). The principal parameters of the system are thus the salt concentration $c$ and the external field $h$. As was shown in Part I for this setup, there is a non-trivial region in the $(c, h)$-plane where phase separation occurs on a macroscopic scale. Specifically, for $(c, h)$ in this region, a droplet which takes a nontrivial (i.e., non-zero and non-one) fraction of the entire volume appears in the system. (For "liquid" boundary conditions, the droplet is actually an ice crystal.) In "magnetic" terms, for each $h$ there is a unique value of the magnetization which is achieved by keeping part of the system in the liquid, i.e., the plus Ising state, and part in the solid, i.e., the minus Ising state. This is in sharp contrast to what happens in the unperturbed Ising model where a single value of $h$ (namely, $h=0$ ) corresponds to a whole interval of possible magnetizations.

The main objective of the present paper is to investigate the limit of infinitesimal salt concentrations. We will take this to mean the following: In a system of linear size $L$ we will consider the above "mixed" ensemble with concentration $c$ and external field $h$ scaling to zero as the size of the system, $L$, tends to infinity. The goal is to describe the asymptotic properties of the typical spin configurations, particularly with regards to the formation of droplets. The salt marginal will now be of no interest because salt particles are so sparse that any local observable will eventually report that there is no salt at all. 
The main conclusions of this work are summarized as follows. First, in a regular system of volume $V=L^{d}$ of characteristic dimension $L$, the scaling for both the salt concentration and external field is $L^{-1}$. In particular, we should write $h=b L^{-1}$ and $c=\xi L^{-1}$. Second, considering such a system with boundary condition favoring the liquid state and with $h$ and $c$ enjoying the abovementioned scalings, one of three things will happen as $\xi$ sweeps from 0 to infinity:

(1) If $b$ is sufficiently small negative, the system is always in the liquid state.

(2) If $b$ is of intermediate (negative) values, there is a transition, at some $\xi(b)$ from the ice state to the liquid state.

(3) Most dramatically, for larger (negative) values of $b$, there is a region - parametrized by $\xi_{1}(b)<\xi<\xi_{2}(b)$ - where (macroscopic) phase separation occurs. Specifically, the system holds a large crystalline chunk of ice, whose volume fraction varies from unity to some positive amount as $\xi$ varies from $\xi_{1}(b)$ to $\xi_{2}(b)$. At $\xi=\xi_{2}(b)$, all of the remaining ice suddenly melts.

We obtain analogous results when the boundary conditions favor the ice state, with the ice crystal replaced by a liquid "brine pocket." However, here a new phenomenon occurs: For certain choices of system parameters, the (growing) volume fraction occupied by the brine pocket remains bounded away from one as $\xi$ increases from $\xi_{1}(b)$ to $\xi_{2}(b)$, and then jumps discontinuously to one at $\xi_{2}(b)$. In particular, there are two droplet transitions (see Fig. 1).

Thus, we claim that the onset of freezing point depression is, in fact, a surface phenomenon. Indeed, for very weak solutions, the bulk behavior of the system is determined by a delicate balance between surface order deviations of the temperature and salt concentrations. In somewhat poetic terms, the predictions of this work are that at the liquid-ice coexistence temperature it is possible to melt a substantial portion of the ice via a pinch of salt whose size is only of the order $V^{1-\frac{1}{d}}$. (However, we make no claims as to how long one would have to wait in order to observe this phenomenon.)

The remainder of this paper is organized as follows. In the next section we reiterate the basic setup of our model and introduce some further objects of relevance. The main results are stated in Sections 2.1-2.3; the corresponding proofs come in Section 3. In order to keep the section and formula numbering independent of Part I; we will prefix the numbers from Part I by "I." 

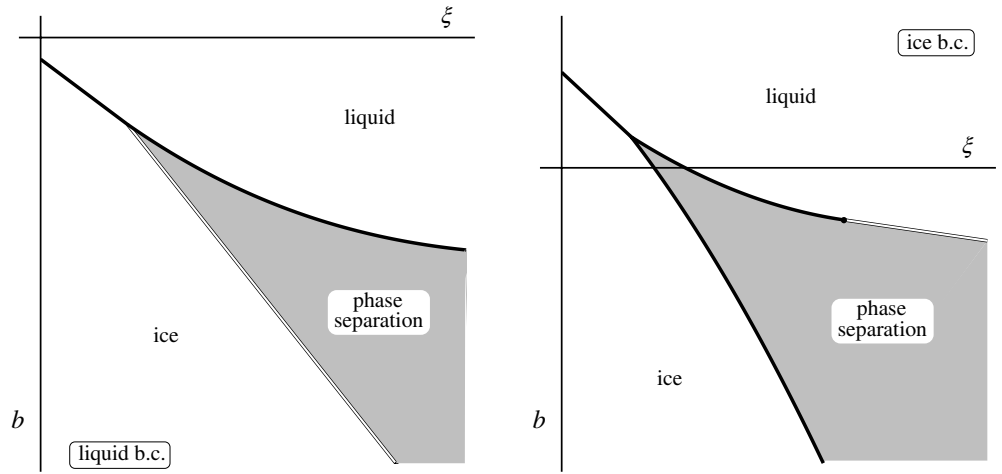

Fig. 1. The phase diagram of the ice-water system with Hamiltonian (1.1) and fixed salt concentration $c$ in a Wulff-shaped vessel of linear size $L$. The left plot corresponds to the system with plus boundary conditions, concentration $c=\xi / L$ and field parameter $h=b / L$, the plot on the right depicts the situation for minus boundary conditions. It is noted that as $\xi$ ranges in $(0, \infty)$ with $b$ fixed, three distinct modes of behavior emerge, in the $L \rightarrow \infty$ limit, depending on the value of $b$. The thick black lines mark the phase boundaries where a droplet transition occurs; on the white lines the fraction of liquid (or solid) in the system changes continuously.

\subsection{Basic Objects}

We begin by a quick reminder of the model; further details and motivation are to be found in Part I. Let $\Lambda \subset \mathbb{Z}^{d}$ be a finite set and let $\partial \Lambda$ denote its (external) boundary. For each $x \in \Lambda$, we introduce the water and salt variables, $\sigma_{x} \in\{-1,+1\}$ and $S_{x} \in\{0,1\}$; on $\partial \Lambda$ we will consider a fixed configuration $\sigma_{\partial \Lambda} \in\{-1,+1\}^{\partial \Lambda}$. The finite-volume Hamiltonian is then a function of $\left(\sigma_{\Lambda}, S_{\Lambda}\right)$ and the boundary condition $\sigma_{\partial \Lambda}$ that takes the form

$$
\beta \mathscr{H}_{\Lambda}\left(\sigma_{\Lambda}, S_{\Lambda} \mid \sigma_{\partial \Lambda}\right)=-J \sum_{\substack{\langle x, y\rangle \\ x \in \Lambda, y \in \mathbb{Z}^{d}}} \sigma_{x} \sigma_{y}-h \sum_{x \in \Lambda} \sigma_{x}+\kappa \sum_{x \in \Lambda} S_{x} \frac{1-\sigma_{x}}{2} .
$$

Here, as usual, $\langle x, y\rangle$ denotes a nearest-neighbor pair on $\mathbb{Z}^{d}$ and the parameters $J, \kappa$ and $h$ represent the chemical affinity of water to water, negative affinity of salt to ice and the difference of the chemical potentials for liquid-water and ice, respectively.

The a priori probability distribution of the pair $\left(\sigma_{\Lambda}, S_{\Lambda}\right)$ takes the usual Gibbs-Boltzmann form $P_{\Lambda}^{\sigma_{\partial \Lambda}}\left(\sigma_{\Lambda}, S_{\Lambda}\right) \propto e^{-\beta \mathscr{H}_{\Lambda}\left(\sigma_{\Lambda}, S_{\Lambda} \mid \sigma_{\partial \Lambda}\right)}$. For reasons explained in Part I, we will focus our attention on the ensemble with a fixed total amount of salt. The relevant quantity is defined by 


$$
N_{\Lambda}=\sum_{x \in \Lambda} S_{x}
$$

The main object of interest in this paper is then the conditional measure

$$
P_{\Lambda}^{\sigma_{\partial \Lambda}, c, h}(\cdot)=P_{\Lambda}^{\sigma_{\partial \Lambda}}\left(\cdot \mid N_{\Lambda}=\lfloor c|\Lambda|\rfloor\right),
$$

where $|\Lambda|$ denotes the number of sites in $\Lambda$. We will mostly focus on the situations when $\sigma_{\partial \Lambda} \equiv+1$ or $\sigma_{\partial \Lambda} \equiv-1$, i.e., the plus or minus boundary conditions. In these cases we denote the above measure by $P_{\Lambda}^{ \pm, c, h}$, respectively.

The surface nature of the macroscopic phase separation - namely, the cases when the concentration scales like the inverse linear scale of the system - indicates that the quantitative aspects of the analysis may depend sensitively on the shape of the volume in which the model is studied. Thus, to keep this work manageable, we will restrict our rigorous treatment of these cases to volumes of a particular shape in which the droplet cost is the same as in infinite volume. The obvious advantage of this restriction is the possibility of explicit calculations; the disadvantage is that the shape actually depends on the value of the coupling constant $J$. Notwithstanding, we expect that all of our findings are qualitatively correct even in rectangular volumes but that cannot be guaranteed without a fair amount of extra work; see ref. 17 for an example.

Let $V \subset \mathbb{R}^{d}$ be a connected set with connected complement and unit Lebesgue volume. We will consider a sequence $\left(V_{L}\right)$ of lattice volumes which are just discretized blow-ups of $V$ by scale factor $L$ :

$$
V_{L}=\left\{x \in \mathbb{Z}^{d}: x / L \in V\right\} .
$$

(The sequence of $L \times \cdots \times L$ boxes $\left(\Lambda_{L}\right)$ from Part I is recovered by letting $V=[0,1)^{d}$.) The particular "shape" $V$ for which we will prove the macroscopic phase separation coincides with that of an equilibrium droplet - the Wulff-shaped volume - which we will define next. We will stay rather succinct; details and proofs can be found in standard literature on Wulff construction (refs. 2, 4, 5, 7, 8, 12 or the review 6). Readers familiar with these concepts may consider skipping the rest of this section and passing directly to the statements of our main results.

Consider the ferromagnetic Ising model at coupling $J \geqslant 0$ and zero external field and let $\mathbb{P}_{\Lambda}^{ \pm, J}$ denote the corresponding Gibbs measure in finite volume $\Lambda \subset \mathbb{Z}^{d}$ and plus/minus boundary conditions. As is well known, there exists a number $J_{\mathrm{c}}=J_{\mathrm{c}}(d)$, with $J_{\mathrm{c}}(1)=\infty$ and $J_{\mathrm{c}}(d) \in(0, \infty)$ 
if $d \geqslant 2$, such that for every $J>J_{\mathrm{c}}$ the expectation of any spin in $\Lambda$ with respect to $\mathbb{P}_{\Lambda}^{ \pm, J}$ is bounded away from zero uniformly in $\Lambda \subset \mathbb{Z}^{d}$. The limiting value of this expectation in the plus state - typically called the spontaneous magnetization - will be denoted by $m_{\star}=m_{\star}(J)$. (Note that $m_{\star}=0$ for $J<J_{\mathrm{c}}$ while $m_{\star}>0$ for $J>J_{\mathrm{c}}$.)

Next we will recall the basic setup for the analysis of surface phenomena. For each unit vector $\mathbf{n} \in \mathbb{R}^{d}$, we first define the surface free energy $\tau_{J}(\mathbf{n})$ in direction $\mathbf{n}$. To this end let us consider a rectangular box $V(N, M) \subset \mathbb{R}^{d}$ with "square" base of side $N$ and height $M$ oriented such that $\mathbf{n}$ is orthogonal to the base. The box is centered at the origin. We let $Z_{N, M}^{+, J}$ denote the Ising partition function in $V(N, M) \cap \mathbb{Z}^{d}$ with plus boundary conditions. We will also consider the inclined Dobrushin boundary condition which takes value +1 at the sites $x$ of the boundary of $V(N, M) \cap \mathbb{Z}^{d}$ for which $x \cdot \mathbf{n}>0$ and -1 at the other sites. Denoting the corresponding partition function by $Z_{N, M}^{ \pm, J, \mathbf{n}}$, the surface free energy $\tau_{J}(\mathbf{n})$ is then defined by

$$
\tau_{J}(\mathbf{n})=-\lim _{M \rightarrow \infty} \lim _{N \rightarrow \infty} \frac{1}{N^{d-1}} \log \frac{Z_{N, M}^{ \pm, J, \mathbf{n}}}{Z_{N, M}^{+, J}} .
$$

The limit exists by subadditivity arguments. The quantity $\tau_{J}(\mathbf{n})$ determines the cost of an interface orthogonal to vector $\mathbf{n}$.

As expected, as soon as $J>J_{\mathrm{c}}$, the function $\mathbf{n} \mapsto \tau_{J}(\mathbf{n})$ is uniformly positive. ${ }^{(14)}$ In order to evaluate the cost of a curved interface, $\tau_{J}(\mathbf{n})$ will have to be integrated over the surface. Explicitly, we will let $J>J_{\mathrm{c}}$ and, given a bounded set $V \subset \mathbb{R}^{d}$ with piecewise smooth boundary, we define the Wulff functional $\mathscr{W}_{J}$ by the integral

$$
\mathscr{W}_{J}(V)=\int_{\partial V} \tau_{J}(\mathbf{n}) d A,
$$

where $d A$ is the (Hausdorff) surface measure and $\mathbf{n}$ is the positiondependent unit normal vector to the surface. The Wulff shape $W$ is the unique minimizer (modulo translation) of $V \mapsto \mathscr{W}(V)$ among bounded sets $V \subset \mathbb{R}^{d}$ with piecewise smooth boundary and unit Lebesgue volume. We let $\left(W_{L}\right)$ denote the sequence of Wulff-shaped lattice volumes defined from $V=W$ via (1.5).

\section{MAIN RESULTS}

We are now in a position to state and prove our main results. As indicated before, we will focus on the limit of infinitesimal concentrations 
(and external fields) where $c$ and $h$ scale as the reciprocal of the linear size of the system. Our results come in four theorems: In Theorem 2.1 we state the basic surface-order large-deviation principle. Theorems 2.2 and 2.3 describe the minimizers of the requisite rate functions for liquid and ice boundary conditions, respectively. Finally, Theorem 2.4 provides some control of the spin marginal of the corresponding Gibbs measure.

\subsection{Large Deviation Principle for Magnetization}

The control of the regime under consideration involves the surface-order large-deviation principle for the total magnetization in the Ising model. In a finite set $\Lambda \subset \mathbb{Z}^{d}$, the quantity under considerations is given by

$$
M_{\Lambda}=\sum_{x \in \Lambda} \sigma_{x}
$$

Unfortunately, the rigorous results available at present for $d \geqslant 3$ do not cover all of the cases to which our analysis might apply. In order to reduce the amount of necessary provisos in the statement of the theorems, we will formulate the relevant properties as an assumption:

Assumption A. Let $d \geqslant 2$ and let us consider a sequence of Wulffshape volumes $W_{L}$. Let $J>J_{\mathrm{c}}$ and recall that $\mathbb{P}_{W_{L}}^{ \pm, J}$ denotes the Gibbs state of the Ising model in $W_{L}$, with \pm -boundary condition and coupling constant $J$. Let $m_{\star}=m_{\star}(J)$ denote the spontaneous magnetization. Then there exist functions $\mathscr{M}_{ \pm, J}:\left[-m_{\star}, m_{\star}\right] \rightarrow[0, \infty)$ such that

$$
\lim _{\epsilon \downarrow 0} \lim _{L \rightarrow \infty} \frac{1}{L^{d-1}} \log \mathbb{P}_{L}^{ \pm, J}\left(\left|M_{L}-m L^{d}\right| \leqslant \epsilon L^{d}\right)=-\mathscr{M}_{ \pm, J}(m)
$$

holds for each $m \in\left[-m_{\star}, m_{\star}\right]$. Moreover, there is a constant $w_{1} \in(0, \infty)$ such that

$$
\mathscr{M}_{ \pm, J}(m)=\left(\frac{m_{\star} \mp m}{2 m_{\star}}\right)^{\frac{d-1}{d}} w_{1}
$$

is true for all $m \in\left[-m_{\star}, m_{\star}\right]$.

The first part of Assumption A - the surface-order large-deviation principle (2.2) - has rigorously been verified for square boxes (and magnetizations near $\left.\pm m_{\star}\right)$ in $d=2^{(8,12)}$ and in $d \geqslant 3$. $^{(5,7)}$ The extension to Wulff-shape domains for all $m \in\left[-m_{\star}, m_{\star}\right]$ requires only minor modifications in $d=2 .^{(16)}$ For $d \geqslant 3$ Wulff-shape domains should be analogously 
controllable but explicit details have not appeared. The fact (proved in ref. 16 for $d=2$ ) that the rate function is given by (2.3) for all magnetizations in $\left[-m_{\star}, m_{\star}\right]$ is specific to the Wulff-shape domains; for other domains one expects the formula to be true only when $\left|m_{\star} \mp m\right|$ is small enough to ensure that the appropriately-sized Wulff-shape droplet fits inside the enclosing volume. Thus, Assumption $\mathrm{A}$ is a proven fact for $d=2$, and it is imminently provable for $d \geqslant 3$.

The underlying reason why (2.2) holds is the existence of multiple states. Indeed, to achieve the magnetization $m \in\left(-m_{\star}, m_{\star}\right)$ one does not have to alter the local distribution of the spin configurations (which is what has to be done for $\left.m \notin\left[-m_{\star}, m_{\star}\right]\right)$; it suffices to create a droplet of one phase inside the other. The cost is just the surface free energy of the droplet; the best possible droplet is obtained by optimizing the Wulff functional (1.7). This is the content of (2.3). However, the droplet is confined to a finite set and, once it becomes sufficiently large, the shape of the enclosing volume becomes relevant. In generic volumes the presence of this additional constraint in the variational problem actually makes the resulting cost larger than (2.3) - which represents the cost of an unconstrained droplet. But, in Wulff-shape volumes, (2.3) holds regardless of the droplet size as long as $|m| \leqslant m_{\star}$. An explicit formula for $\mathscr{M}_{ \pm, J}(m)$ for square volumes has been obtained in $d=2^{(17)}$; the situation in $d \geqslant 3$ has been addressed in refs. 10 and 11 .

On the basis of the above assumptions, we are ready to state our first main result concerning the measure $P_{W_{L}}^{ \pm, c h}$ with $c \sim \xi / L$ and $h \sim b / L$. Using $\theta$ to denote the fraction of salt on the plus spins, we begin by introducing the relevant entropy function

$$
\Upsilon(m, \theta)=-\theta \log \frac{2 \theta}{1+m}-(1-\theta) \log \frac{2(1-\theta)}{1-m} .
$$

We remark that if we write a full expression for the bulk entropy, $\Xi(m, \theta ; c)$, see formula (3.5), at fixed $m, c$ and $\theta$, then, modulo some irrelevant terms, the quantity $\Upsilon(m, \theta)$ is given by $(\partial / \partial c) \Xi(m, \theta ; c)$ at $c=0$. Thus, when we scale $c \sim \xi / L$, the quantity $\xi \Upsilon(m, \theta)$ represents the relevant (surface order) entropy of salt with $m$ and $\theta$ fixed. The following is an analogue of Theorem I.2.1 from Part I for the case at hand:

Theorem 2.1. Let $d \geqslant 2$ and let $J>J_{\mathrm{c}}(d)$ and $\kappa>0$ be fixed. Let $m_{\star}=m_{\star}(J)$ denote the spontaneous magnetization of the Ising model. Suppose that (2.2) in Assumption A holds and let $\left(c_{L}\right)$ and $\left(h_{L}\right)$ be two sequences such that $c_{L} \geqslant 0$ for all $L$ and that the limits

$$
\xi=\lim _{L \rightarrow \infty} L c_{L} \quad \text { and } \quad b=\lim _{L \rightarrow \infty} L h_{L}
$$


exist and are finite. Then for all $m \in\left[-m_{\star}, m_{\star}\right]$,

$$
\begin{gathered}
\lim _{\epsilon \downarrow 0} \lim _{L \rightarrow \infty} \frac{1}{L^{d-1}} \log P_{W_{L}}^{ \pm, c_{L}, h_{L}}\left(\left|M_{L}-m L^{d}\right| \leqslant \epsilon L^{d}\right) \\
=-Q_{b, \xi}^{ \pm}(m)+\inf _{\left|m^{\prime}\right| \leqslant m_{\star}} Q_{b, \xi}^{ \pm}\left(m^{\prime}\right),
\end{gathered}
$$

where $Q_{b, \xi}^{ \pm}(m)=\inf _{\theta \in[0,1]} \mathscr{Q}_{b, \xi}^{ \pm}(m, \theta)$ with

$$
\mathscr{Q}_{b, \xi}^{ \pm}(m, \theta)=-b m-\xi \kappa \theta-\xi \Upsilon(m, \theta)+\mathscr{M}_{ \pm, J}(m),
$$

Various calculations in the future will require a somewhat more explicit expression for the rate function $m \mapsto Q_{b, \xi}^{ \pm}(m)$ on the right-hand side of (2.6). To derive such an expression, we first note that the minimizer of $\theta \mapsto \mathscr{Q}_{b, \xi}^{ \pm}(m, \theta)$ is uniquely determined by the equation

$$
\frac{\theta}{1-\theta}=\frac{1+m}{1-m} e^{\kappa}
$$

Plugging this into $\mathscr{2}_{b, \xi}^{ \pm}(m, \theta)$ tells us that

$$
Q_{b, \xi}^{ \pm}(m)=-b m-\xi g(m)+\mathscr{M}_{ \pm, J}(m)
$$

where

$$
g(m)=\log \left(\frac{1-m}{2}+e^{\kappa} \frac{1+m}{2}\right) .
$$

Clearly, $g$ is strictly concave for any $\kappa>0$.

\subsection{Macroscopic Phase Separation - "Liquid" Boundary Conditions}

While Theorem I.2.1 of Part I and Theorem 2.1 above may appear formally similar, the solutions of the associated variational problems are rather different. Indeed, unlike the "bulk" rate function $G_{h, c}(m)$ of Part I, the functions $Q_{b, \xi}^{ \pm}(m)$ are not generically strictly convex which in turns leads to a possibility of having more than one minimizing $m$. We consider first the case of plus (that is, liquid water) boundary conditions. 
Let $d \geqslant 2$ and let $J>J_{\mathrm{c}}(d)$ and $\kappa>0$ be fixed. To make our formulas manageable, for any function $\phi:\left[-m_{\star}, m_{\star}\right] \rightarrow \mathbb{R}$ let us use the abbreviation

$$
D_{\phi}^{\star}=\frac{\phi\left(m_{\star}\right)-\phi\left(-m_{\star}\right)}{2 m_{\star}}
$$

for the slope of $\phi$ between $-m_{\star}$ and $m_{\star}$. Further, let us introduce the quantity

$$
\xi_{\mathrm{t}}=\frac{w_{1}}{2 m_{\star} d}\left(g^{\prime}\left(-m_{\star}\right)-D_{g}^{\star}\right)^{-1}
$$

and the piecewise linear function $b_{2}:[0, \infty) \rightarrow \mathbb{R}$ which is defined by

$$
b_{2}(\xi)= \begin{cases}-\frac{w_{1}}{2 m_{\star}}-\xi D_{g}^{\star}, & \xi<\xi_{\mathrm{t}} \\ -\frac{d-1}{d} \frac{w_{1}}{2 m_{\star}}-\xi g^{\prime}\left(-m_{\star}\right), & \xi \geqslant \xi_{\mathrm{t}} .\end{cases}
$$

Our next result is as follows:

Theorem 2.2. Let $d \geqslant 2$ and let $J>J_{\mathrm{c}}(d)$ and $\kappa>0$ be fixed. Let the objects $Q_{b, \xi}^{+}, \xi_{\mathrm{t}}$ and $b_{2}$ be as defined above. Then there exists a (strictly) decreasing and continuous function $b_{1}:[0, \infty) \rightarrow \mathbb{R}$ with the following properties:

(1) $b_{1}(\xi) \geqslant b_{2}(\xi)$ for all $\xi \geqslant 0$, and $b_{1}(\xi)=b_{2}(\xi)$ iff $\xi \leqslant \xi_{\text {t }}$.

(2) $b_{1}^{\prime}$ is continuous on $[0, \infty), b_{1}^{\prime}(\xi) \rightarrow-g^{\prime}\left(m_{\star}\right)$ as $\xi \rightarrow \infty$ and $b_{1}$ is strictly convex on $\left[\xi_{\mathrm{t}}, \infty\right)$.

(3) For $b \neq b_{1}(\xi), b_{2}(\xi)$, the function $m \mapsto Q_{b, \xi}^{+}(m)$ is minimized by a single number $m=m_{+}(b, \xi) \in\left[-m_{\star}, m_{\star}\right]$ which satisfies

$$
m_{+}(b, \xi) \begin{cases}=m_{\star}, & \text { if } b>b_{1}(\xi), \\ \in\left(-m_{\star}, m_{\star}\right), & \text { if } b_{2}(\xi)<b<b_{1}(\xi), \\ =-m_{\star}, & \text { if } b<b_{2}(\xi) .\end{cases}
$$

(4) The function $b \mapsto m_{+}(b, \xi)$ is strictly increasing for $b \in$ $\left[b_{2}(\xi), b_{1}(\xi)\right]$, is continuous on the portion of the line $b=b_{2}(\xi)$ for which $\xi>\xi_{\text {t }}$ and has a jump discontinuity along the line defined by $b=b_{1}(\xi)$. The only minimizers at $b=b_{1}(\xi)$ and $b=b_{2}(\xi)$ are the corresponding limits of $b \mapsto m_{+}(b, \xi)$. 
The previous statement essentially characterizes the phase diagram for the cases described in (2.5). Focusing on the plus boundary condition we have the following facts: For reduced concentrations $\xi$ exceeding the critical value $\xi_{\mathrm{t}}$, there exists a range of reduced magnetic fields $b$ where a non-trivial droplet appears in the system. This range is enclosed by two curves which are the graphs of functions $b_{1}$ and $b_{2}$ above. For $b$ decreasing to $b_{1}(\xi)$, the system is in the pure plus - i.e., liquid - phase but, interestingly, at $b_{1}$ a macroscopic droplet - an ice crystal - suddenly appears in the system. As $b$ further decreases the ice crystal keeps growing to subsume the entire system when $b=b_{2}(\xi)$. For $\xi \leqslant \xi_{\text {t }}$ no phase separation occurs; the transition at $b=b_{1}(\xi)=b_{2}(\xi)$ is directly from $m=m_{\star}$ to $m=$ $-m_{\star}$.

It is noted that the situation for $\xi$ near zero corresponds to the Ising model with negative external field proportional to $1 / L$. In two-dimensional setting, the latter problem has been studied in ref. 16. As already mentioned, the generalizations to rectangular boxes will require a non-trivial amount of extra work. For the unadorned Ising model (i.e., $c=0$ ) this has been carried out in great detail in ref. 17 for $d=2$ (see also ref. 13) and in less detail in general dimensions. ${ }^{(10,11)}$

It is reassuring to observe that the above results mesh favorably with the corresponding asymptotic of Part I. For finite concentrations and external fields, there are two curves, $c \mapsto h_{+}(c)$ and $c \mapsto h_{-}(c)$, which mark the boundaries of the phase separation region against the liquid and ice regions, respectively. The curve $c \mapsto h_{+}(c)$ is given by the equation

$$
h_{+}(c)=\frac{1}{2} \log \frac{1-q_{+}}{1-q_{-}},
$$

where $\left(q_{+}, q_{-}\right)$is the (unique) solution of

$$
\frac{q_{+}}{1-q_{+}}=e^{\kappa} \frac{q_{-}}{1-q_{-}}, \quad q_{+} \frac{1+m_{\star}}{2}+q_{-} \frac{1-m_{\star}}{2}=c .
$$

The curve $c \mapsto h_{-}(c)$ is defined by the same equations with the roles of $m_{\star}$ and $-m_{\star}$ interchanged. Since $h_{ \pm}(0)=0$, these can be linearized around the point $(0,0)$. Specifically, plugging $b / L$ for $h$ and $\xi / L$ for $c$ into $h=h_{ \pm}(c)$ and letting $L \rightarrow \infty$ yields the linearized versions

$$
b_{ \pm}=h_{ \pm}^{\prime}(0) \xi
$$

of $h_{+}$and $h_{-}$. It is easy to check that $h_{ \pm}^{\prime}(0)=-g^{\prime}\left( \pm m_{\star}\right)$ and so, in the limit $\xi \rightarrow \infty$, the linear function $b_{+}$has the same slope as $b_{1}$ while $b_{-}$ 
has the same slope as $b_{2}$ above. Theorem 2.2 gives a detailed description of how these linearized curves ought to be continued into (infinitesimal) neighborhoods of size $1 / L$ around $(0,0)$.

\subsection{Macroscopic Phase Separation - "Ice" Boundary Conditions}

Next we consider minus (ice) boundary conditions, where the requisite liquid water, phase separation and ice regions will be defined using the functions $\tilde{b}_{1} \geqslant \tilde{b}_{2}$. As for the plus boundary conditions, there is a value $\tilde{\xi}_{\mathrm{t}}>0$ where the phase separation region begins, but now we have a new phenomenon: For some (but not all) choices of $J$ and $\kappa$, there exists a nonempty interval $\left(\tilde{\xi}_{\mathrm{t}}, \tilde{\xi}_{\mathrm{u}}\right)$ of $\xi$ for which two distinct droplet transitions occur. Specifically, as $b$ increases, the volume fraction occupied by the droplet first jumps discontinuously at $\tilde{b}_{2}(\xi)$ from zero to a strictly positive value, then increases but stays bounded away from one, and then, at $b=\tilde{b}_{1}(\xi)$, jumps discontinuously to one; i.e., the ice surrounding the droplet suddenly melts.

For each $J>J_{\mathrm{c}}(d)$ and each $\kappa$, consider the auxiliary quantities

$$
\xi_{1}=\frac{w_{1}}{2 m_{\star} d}\left(D_{g}^{\star}-g^{\prime}\left(m_{\star}\right)\right)^{-1} \quad \text { and } \quad \xi_{2}=-\frac{(d-1) w_{1}}{\left(2 m_{\star} d\right)^{2} g^{\prime \prime}\left(m_{\star}\right)}
$$

(Note that, due to the concavity property of $g$, both $\xi_{1}$ and $\xi_{2}$ are finite and positive.) The following is a precise statement of the above:

Theorem 2.3. Let $d \geqslant 2$ and let $J>J_{\mathrm{c}}(d)$ and $\kappa>0$ be fixed. Then there exist two (strictly) decreasing and continuous functions $\tilde{b}_{1}, \tilde{b}_{2}$ : $[0, \infty) \rightarrow \mathbb{R}$ and numbers $\tilde{\xi}_{\mathrm{t}}, \widetilde{\xi}_{\mathrm{u}} \in(0, \infty)$ with $\tilde{\xi}_{\mathrm{t}} \leqslant \tilde{\xi}_{\mathrm{u}}$ such that the following properties hold:

(1) $\tilde{b}_{1}(\xi) \geqslant \tilde{b}_{2}(\xi)$ for all $\xi \geqslant 0$, and $\tilde{b}_{1}(\xi)=\tilde{b}_{2}(\xi)$ iff $\xi \leqslant \tilde{\xi}_{\mathrm{t}}$.

(2) $\tilde{b}_{2}$ is strictly concave on $\left[\tilde{\xi}_{\mathrm{t}}, \infty\right), \tilde{b}_{2}^{\prime}(\xi) \rightarrow-g^{\prime}\left(-m_{\star}\right)$ as $\xi \rightarrow \infty, \tilde{b}_{1}$ is strictly convex on $\left(\tilde{\xi}_{\mathrm{t}}, \tilde{\xi}_{\mathrm{u}}\right)$ and, outside this interval,

$$
\tilde{b}_{1}(\xi)= \begin{cases}\frac{w_{1}}{2 m_{\star}}-\xi D_{g}^{\star}, & \xi \leqslant \tilde{\xi}_{\mathrm{t}}, \\ \frac{d-1}{d} \frac{w_{1}}{2 m_{\star}}-\xi g^{\prime}\left(m_{\star}\right), & \xi \geqslant \tilde{\xi}_{\mathrm{u}} .\end{cases}
$$

(3a) If $\xi_{1} \geqslant \xi_{2}$, then $\tilde{\xi}_{\mathrm{t}}=\tilde{\xi}_{\mathrm{u}}=\xi_{1}$ and $\tilde{b}_{2}^{\prime}$ is continuous on $[0, \infty)$. 
(3b) If $\xi_{1}<\xi_{2}$ then $\tilde{\xi}_{\mathrm{t}}<\xi_{1}<\tilde{\xi}_{\mathrm{u}}=\xi_{2}$ and neither $b_{1}^{\prime}$ nor $b_{2}^{\prime}$ is continuous at $\tilde{\xi}_{\mathrm{t}}$. Moreover, there exists $m_{0} \in\left(-m_{\star}, m_{\star}\right)$ such that, as $\xi_{\downarrow} \downarrow \tilde{\xi}_{\mathrm{t}}$,

$$
b_{1}^{\prime}(\xi) \rightarrow-\frac{g\left(m_{\star}\right)-g\left(m_{0}\right)}{m_{\star}-m_{0}} \quad \text { and } \quad b_{2}^{\prime}(\xi) \rightarrow-\frac{g\left(m_{0}\right)-g\left(-m_{\star}\right)}{m_{0}+m_{\star}}
$$

(4) For $b \neq \tilde{b}_{1}(\xi), \tilde{b}_{2}(\xi)$, the function $m \mapsto Q_{b, \xi}^{-}(m)$ is minimized by a single number $m=m_{-}(b, \xi) \in\left[-m_{\star}, m_{\star}\right]$ which satisfies

$$
m_{-}(b, \xi) \begin{cases}=m_{\star}, & \text { if } b>\tilde{b}_{1}(\xi), \\ \in\left(-m_{\star}, m_{\star}\right), & \text { if } \tilde{b}_{2}(\xi)<b<\tilde{b}_{1}(\xi), \\ =-m_{\star}, & \text { if } b<\tilde{b}_{2}(\xi) .\end{cases}
$$

(5) The function $b \mapsto m_{-}(b, \xi)$ is strictly increasing in $b$ for $b \in$ $\left[\tilde{b}_{2}(\xi), \tilde{b}_{1}(\xi)\right]$, is continuous on the portion of the line $b=\tilde{b}_{1}(\xi)$ for which $\xi \geqslant \widetilde{\xi}_{\text {u }}$ and has jump discontinuities both along the line defined by $b=\tilde{b}_{2}(\xi)$ and along the portion of the line $b=\tilde{b}_{1}(\xi)$ for which $\tilde{\xi}_{\mathrm{t}}<\xi<$ $\tilde{\xi}_{\mathrm{u}}$. There are two minimizers at the points where $b \mapsto m_{-}(b, \xi)$ is discontinuous with the exception of $(b, \xi)=\left(\tilde{b}_{1}\left(\tilde{\xi}_{\mathrm{t}}\right), \tilde{\xi}_{\mathrm{t}}\right)=\left(\tilde{b}_{2}\left(\tilde{\xi}_{\mathrm{t}}\right), \tilde{\xi}_{\mathrm{t}}\right)$ when $\tilde{\xi}_{\mathrm{t}}<\tilde{\xi}_{\mathrm{u}}$, where there are three minimizers; namely, $\pm m_{\star}$ and $m_{0}$ from part (3b).

As a simple consequence of the definitions, it is seen that the question of whether or not $\xi_{1} \geqslant \xi_{2}$ is equivalent to the question whether or not

$$
g\left(m_{\star}\right)-2 m_{\star} g^{\prime}\left(m_{\star}\right)+\frac{d}{d-1}\left(2 m_{\star}\right)^{2} g^{\prime \prime}\left(m_{\star}\right) \leqslant g\left(-m_{\star}\right) .
$$

We claim that (2.22) will hold, or fail, depending on the values of the various parameters of the model. Indeed, writing $\epsilon=\tanh (\kappa / 2)$ we get

$$
g(m)=\log (1+\epsilon m)+\text { const. }
$$

Regarding the quantity $\epsilon m$ as a "small parameter," we easily verify that the desired inequality holds to the lowest non-vanishing order. Thus, if $m_{\star}$ is small enough, then (2.22) holds for all $\kappa$, while it is satisfied for all $m_{\star}$ whenever $\kappa$ is small enough. On the other hand, as $\kappa$ tends to infinity, $g\left(m_{\star}\right)-g\left(-m_{\star}\right)$ tends to $\log \frac{1+m_{\star}}{1-m_{\star}}$, while the various relevant derivatives of $g$ are bounded independently of $m_{\star}$. Thus, as $m_{\star} \rightarrow 1$, which happens when $J \rightarrow \infty$, the condition (2.22) is violated for $\kappa$ large enough. Evidently, the gap $\tilde{\xi}_{\mathrm{u}}-\tilde{\xi}_{\mathrm{t}}$ is strictly positive for some choices of $J$ and $\kappa$, and vanishes for others. 
Since $\tilde{b}_{1}(0)>0$, for $\xi$ sufficiently small the ice region includes points with $b>0$. Let us also show that the phase separation region can rise above $b=0$; as indicated in the plot on the right of Fig. 1. Clearly, it suffices to consider $b=0$ and establish that for some $J, \kappa$ and $\xi$, the absolute minimum of $m \mapsto Q_{0, \xi}^{-}(m)$ does not occur at $\pm m_{\star}$. This will certainly hold if

$$
\left(Q_{0, \xi}^{-}\right)^{\prime}\left(m_{\star}\right)>0 \quad \text { and } \quad Q_{0, \xi}^{-}\left(-m_{\star}\right)>Q_{0, \xi}^{-}\left(m_{\star}\right)
$$

or, equivalently, if

$$
\frac{d-1}{d} \frac{w_{1}}{2 m_{\star}}>\xi g^{\prime}\left(m_{\star}\right) \quad \text { and } \quad \xi\left(g\left(m_{\star}\right)-g\left(-m_{\star}\right)\right)>w_{1}
$$

are both true. Some simple algebra shows that the last inequalities hold for some $\xi$ once

$$
\frac{d-1}{d}\left(g\left(m_{\star}\right)-g\left(-m_{\star}\right)\right)>2 m_{\star} g^{\prime}\left(m_{\star}\right) .
$$

But, as we argued a moment ago, the difference $g\left(m_{\star}\right)-g\left(-m_{\star}\right)$ can be made arbitrary large by taking $\kappa \gg 1$ and $m_{\star}$ sufficiently close to one, while $g^{\prime}\left(m_{\star}\right)$ is bounded in these limits. So, indeed, the phase separation region pokes above the $b=0$ axis once $\kappa \gg 1$ and $J \gg 1$.

Comparing to the linear asymptotic of the phase diagram from Part I, we see that in the finite-volume system with minus (ice) boundary condition, the lines bounding the phase separation region are shifted upward and again are pinched together. In this case it is the line $b=\tilde{b}_{1}(\xi)$ that is parallel to its counterpart $b=h_{+}^{\prime}(0) \xi$ for $\xi>\tilde{\xi}_{\mathrm{u}}$, while $b=\tilde{b}_{2}(\xi)$ has the same asymptotic slope (in the limit $\xi \rightarrow \infty$ ) as the function $b=h_{-}^{\prime}(0) \xi$.

\subsection{Properties of the Spin Marginal}

On the basis of Theorems 2.1-2.4, we can now provide a routine characterization of the typical configurations in measure $P_{W_{L}}^{ \pm, c_{L}, h_{L}}$. The following is an analogue of Theorem 2.2 of Part I for the cases at hand:

Theorem 2.4. Let $d \geqslant 2$ and let $J>J_{\mathrm{c}}(d)$ and $\kappa>0$ be fixed. Suppose that Assumption A holds and let $\left(c_{L}\right)$ and $\left(h_{L}\right)$ be two sequences such that $c_{L} \geqslant 0$ for all $L$ and that the limits $\xi$ and $b$ in (2.5) exist and 
are finite. Let us define two sequences of Borel probability measures $\rho_{L}^{ \pm}$ on $\left[-m_{\star}, m_{\star}\right]$ by putting

$$
\rho_{L}^{ \pm}([-1, m])=P_{W_{L}}^{ \pm, c_{L}, h_{L}}\left(M_{L} \leqslant m L^{d}\right), \quad m \in[-1,1]
$$

Then the spin marginal of the measure $P_{W_{L}}^{ \pm, c_{L}, h_{L}}$ can again be written as a convex combination of the Ising measures with fixed magnetization; i.e., for any set $\mathcal{A}$ of configurations $\left(\sigma_{x}\right)_{x \in \Lambda_{L}}$,

$$
P_{W_{L}}^{ \pm, c_{L}, h_{L}}\left(\mathcal{A} \times\{0,1\}^{W_{L}}\right)=\int \rho_{L}^{ \pm}(\mathrm{d} m) \mathbb{P}_{W_{L}}^{ \pm, J}\left(\mathcal{A} \mid M_{L}=\left\lfloor m L^{d}\right\rfloor\right)
$$

Moreover, any (weak) subsequential limit $\rho^{ \pm}$of measures $\rho_{L}^{ \pm}$is concentrated on the minimizers of $m \mapsto Q_{b, \xi}^{ \pm}(m)$. In particular, for $b \neq$ $b_{1}(\xi), b_{2}(\xi)$ the limit $\rho^{+}=\lim _{L \rightarrow \infty} \rho_{L}^{+}$exists and is simply the Dirac mass at $m_{+}(b, \xi)$ - the quantity from Theorem 2.2 - and similarly for $\rho^{-}=$ $\lim _{L \rightarrow \infty} \rho_{L}^{-}$and $b \neq \tilde{b}_{1}(\xi), \tilde{b}_{2}(\xi)$.

On the basis of Theorems 2.1-2.4, we can draw the following conclusions: For $d$-dimensional systems of scale $L$ with the total amount of salt proportional to $L^{d-1}$ (i.e., the system boundary), phase separation occurs dramatically in the sense that all of a sudden a non-trivial fraction of the system melts/freezes (depending on the boundary condition). In hindsight, this is perhaps not so difficult to understand. While a perturbation of size $L^{d-1}$ cannot influence the bulk properties of the system with a single phase, here the underlying system is at phase coexistence. Thus the cost of a droplet is only of order $L^{d-1}$, so it is not unreasonable that a comparable amount of salt will cause dramatic effects.

It is worth underscoring that the jump in the size of the macroscopic droplet at $b=b_{1}$ or $b=\tilde{b}_{2}$ decreases with increasing $\xi$. Indeed, in the extreme limit, when the concentration is finite (nonzero) we know that no macroscopic droplet is present at the transition. But, presumably, by analogy with the results of ref. 4 (see also refs. 3 and 15), there will be a mesoscopic droplet - of a particular scaling - appearing at the transition point. This suggests that a host of intermediate mesoscopic scales may be exhibited depending on how $c_{L}$ and $h_{L}$ tend to zero with the ratio $h_{L} / c_{L}$ approximately fixed. These intermediate behaviors are currently being investigated.

\section{PROOFS OF MAIN RESULTS}

The goal of this section is to prove the results stated in Section 2. We begin by stating a generalized large deviation principle for both magnetization and the fraction of salt on the plus spins from which Theorem 2.1 
follows as an easy corollary. Theorem 2.2 is proved in Section 3.2; Theorems 2.3 and 2.4 are proved in Section 3.3.

\subsection{A Generalized Large-deviation Principle}

We will proceed similarly as in the proof of Theorem I.3.7 from Part I. Let $\Lambda \subset \mathbb{Z}^{d}$ be a finite set and let us reintroduce the quantity

$$
Q_{\Lambda}=\sum_{x \in \Lambda} S_{x} \frac{1+\sigma_{x}}{2},
$$

which gives the total amount of salt on the plus spins in $\Lambda$. Recall that $\mathbb{E}_{\Lambda}^{ \pm, J}$ denotes the expectation with respect to the (usual) Ising measure with coupling constant $J$ and plus/minus boundary conditions. First we generalize a couple of statements from Part I:

Lemma 3.1. Let $\Lambda \subset \mathbb{Z}^{d}$ be a finite set. Then for any fixed spin configuration $\bar{\sigma}=\left(\bar{\sigma}_{x}\right) \in\{-1,1\}^{\Lambda}$, all salt configurations $\left(S_{x}\right) \in\{0,1\}^{\Lambda}$ with the same $N_{\Lambda}$ and $Q_{\Lambda}$ have the same probability in the conditional measure $P_{\Lambda}^{ \pm, c, h}(\cdot \mid \sigma=\bar{\sigma})$. Moreover, for any $\bar{S}=\left(\bar{S}_{x}\right) \in\{0,1\}^{\Lambda}$ with $N_{\Lambda}=\lfloor c|\Lambda|\rfloor$ and for any $m \in[-1,1]$,

$$
\begin{aligned}
& P_{\Lambda}^{ \pm, c, h}\left(\bar{S} \text { occurs, } M_{\Lambda}=\lfloor m|\Lambda|\rfloor\right)
\end{aligned}
$$

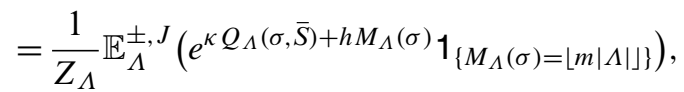

where the normalization constant is given by

$$
Z_{\Lambda}=\sum_{S^{\prime} \in\{0,1\}^{\Lambda}} 1_{\left\{N_{\Lambda}\left(S^{\prime}\right)=\lfloor c|\Lambda|\rfloor\right\}} \mathbb{E}_{\Lambda}^{ \pm, J}\left(e^{\kappa} Q_{\Lambda}\left(\sigma, S^{\prime}\right)+h M_{\Lambda}(\sigma)\right) .
$$

Proof. This is identical to Lemma I.3.3 from Part I.

Next we will sharpen the estimate from Part I concerning the total entropy carried by the salt. Similarly to the object $\mathcal{A}_{L}^{\theta, c}(\sigma)$ from Part I, for each spin configuration $\sigma=\left(\sigma_{x}\right) \in\{-1,1\}^{\Lambda}$ and numbers $\theta, c \in[0,1]$, we introduce the set

$$
\mathcal{A}_{\Lambda}^{\theta, c}(\sigma)=\left\{\left(S_{x}\right) \in\{0,1\}^{\Lambda}: N_{L}=\lfloor c|\Lambda|\rfloor, Q_{L}=\lfloor\theta c|\Lambda|\rfloor\right\} .
$$

Clearly, the size of $\mathcal{A}_{\Lambda}^{\theta, c}(\sigma)$ is the same for all $\sigma$ with a given value of the magnetization; we will thus let $A_{\Lambda}^{\theta, c}(m)$ denote the common value 
of $\left|\mathcal{A}_{\Lambda}^{\theta, c}(\sigma)\right|$ for those $\sigma$ with $M_{\Lambda}(\sigma)=\lfloor m|\Lambda|\rfloor$. Let $\mathscr{S}(p)=p \log p+(1-$ $p) \log (1-p)$ and let us recall the definition of the entropy function

$$
\Xi(m, \theta ; c)=-\frac{1+m}{2} \mathscr{S}\left(\frac{2 \theta c}{1+m}\right)-\frac{1-m}{2} \mathscr{S}\left(\frac{2(1-\theta) c}{1-m}\right)
$$

cf. formula (I.2.7) from Part I. Then we have:

Lemma 3.2. For each $\eta>0$ there exist constants $C_{1}<\infty$ and $L_{0}<$ $\infty$ such that for all finite $\Lambda \subset \mathbb{Z}^{d}$ with $|\Lambda| \geqslant L_{0}^{d}$, all $\theta, c \in[0,1]$ and all $m$ with $|m| \leqslant 1-\eta$ satisfying

$$
\frac{2 \theta c}{1+m} \leqslant 1-\eta \quad \text { and } \quad \frac{2(1-\theta) c}{1-m} \leqslant 1-\eta
$$

we have

$$
\left|\frac{\log A_{\Lambda}^{\theta, c}(m)}{|\Lambda|}-\Xi(m, \theta ; c)\right| \leqslant C_{1} \frac{\log |\Lambda|}{|\Lambda|} .
$$

Proof. The same calculations that were used in the proof of Lemma I.3.4 from Part I give us

$$
A_{\Lambda}^{\theta, c}(m)=\left(\begin{array}{c}
\frac{1}{2}\left(|\Lambda|+M_{\Lambda}\right) \\
Q_{\Lambda}
\end{array}\right)\left(\begin{array}{c}
\frac{1}{2}\left(|\Lambda|-M_{\Lambda}\right) \\
N_{\Lambda}-Q_{\Lambda}
\end{array}\right)
$$

with the substitutions $M_{\Lambda}=\lfloor m|\Lambda|\rfloor$ and $Q_{\Lambda}=\lfloor\theta c|\Lambda|\rfloor$. By (3.6) and $|m| \leqslant$ $1-\eta$, both combinatorial numbers are well defined once $|\Lambda|$ is sufficiently large (this defines $L_{0}$ ). Thus, we can invoke the Stirling approximation and, eventually, we see that the right-hand side of (3.8) equals $\exp \{|\Lambda| \Xi(m, \theta ; c)\}$ times factors which grow or decay at most like a power of $|\Lambda|$. Taking logs and dividing by $|\Lambda|$, this yields (3.7).

Our final preliminary lemma is concerned with the magnetizations outside $\left[-m_{\star}, m_{\star}\right]$ which are (formally) not covered by Assumption A. Recall the sequence of Wulff shapes $W_{L}$ defined at the end of Section 1.2. Note that $W_{L}$ contains, to within boundary corrections, $L^{d}$ sites.

Lemma 3.3. Suppose that $J>J_{\mathrm{c}}$ and let $c_{L}$ and $h_{L}$ be such that $L c_{L}$ and $L h_{L}$ have finite limits as $L \rightarrow \infty$. For each $\epsilon>0$, we have

$$
\lim _{L \rightarrow \infty} \frac{1}{L^{d-1}} \log P_{W_{L}}^{ \pm, c_{L}, h_{L}}\left(\left|M_{W_{L}}\right| \geqslant\left(m_{\star}+\epsilon\right) L^{d}\right)=-\infty .
$$


Proof. This is a simple consequence of the fact that, in the unadorned Ising magnet, the probability in (3.9) is exponentially small in volume - cf. Theorem I.3.1 - and that with $L h_{L}$ and $L c_{L}$ bounded, there will be at most a surface-order correction. A formal proof proceeds as follows: We write

$$
P_{W_{L}}^{ \pm, c_{L}, h_{L}}\left(Q_{L}=\left\lfloor\theta c_{L} L^{d}\right\rfloor, M_{L}=\left\lfloor m L^{d}\right\rfloor\right)=\frac{\widetilde{K}_{L}(m, \theta)}{Y_{L}}
$$

where

$$
\widetilde{K}_{L}(m, \theta)=A_{W_{L}}^{\theta, c_{L}}(m) e^{h_{L}\left\lfloor m L^{d}\right\rfloor+\kappa\left\lfloor\theta c_{L} L^{d}\right\rfloor} \mathbb{P}_{W_{L}}^{ \pm, J}\left(M_{L}=\left\lfloor m L^{d}\right\rfloor\right)
$$

and where $Y_{L}$ is the sum of $\widetilde{K}_{L}\left(m^{\prime}, \theta^{\prime}\right)$ over all relevant values of $m^{\prime}$ and $\theta^{\prime}$. Under the assumption that both $h_{L}$ and $c_{L}$ behave like $O\left(L^{-1}\right)$, the prefactors of the Ising probability can be bounded between $e^{-C L^{d-1}}$ and $e^{C L^{d-1}}$, for some $C<\infty$, uniformly in $\theta$ and $m$. This yields

$$
P_{W_{L}}^{ \pm, c_{L}, h_{L}}\left(\left|M_{W_{L}}\right| \geqslant\left(m_{\star}+\epsilon\right) L^{d}\right) \leqslant e^{C L^{d-1}} \frac{1}{Y_{L}} \mathbb{P}_{W_{L}}^{ \pm, J}\left(\left|M_{W_{L}}\right| \geqslant\left(m_{\star}+\epsilon\right) L^{d}\right) .
$$

The same argument shows us that $Y_{L}$ can be bounded below by $e^{-C L^{d-1}}$ times the probability that $M_{W_{L}}$ is near zero in the Ising measure $\mathbb{P}_{W_{L}}^{ \pm, J}$. In light of $J>J_{\mathrm{c}}$, Assumption A then gives

$$
\liminf _{L \rightarrow \infty} \frac{1}{L^{d-1}} \log Y_{L}>-\infty .
$$

On the other hand, by Theorem I.3.1 (and the remark that follows it) we have that

$$
\lim _{L \rightarrow \infty} \frac{1}{L^{d-1}} \log \mathbb{P}_{W_{L}}^{ \pm, J}\left(\left|M_{W_{L}}\right| \geqslant\left(m_{\star}+\epsilon\right) L^{d}\right)=-\infty
$$

Plugging this into (3.12), the desired claim follows.

We will use the above lemmas to state and prove a generalization of Theorem 2.1. 
Theorem 3.4. Let $d \geqslant 2$ and let $J>J_{\mathrm{c}}(d)$ and $\kappa \geqslant 0$ be fixed. Let $c_{L} \in[0,1]$ and $h_{L} \in \mathbb{R}$ be two sequences such that the limits $\xi$ and $b$ in (2.5) exist and are finite. For each $m \in\left[-m_{\star}, m_{\star}\right]$ and $\theta \in(-1,1)$, let $\widetilde{\mathcal{B}}_{L, \epsilon}=$ $\widetilde{\mathcal{B}}_{L, \epsilon}\left(m, c_{L}, \theta\right)$ be the set of all $(\sigma, S) \in\{-1,1\}^{W_{L}} \times\{0,1\}^{W_{L}}$ for which the bounds

$$
\left|M_{W_{L}}-m L^{d}\right| \leqslant \epsilon L^{d} \quad \text { and } \quad\left|Q_{W_{L}}-\theta c_{L} L^{d}\right| \leqslant \epsilon L^{d-1}
$$

hold. Then

$$
\lim _{\epsilon \downarrow 0} \lim _{L \rightarrow \infty} \frac{\log P_{W_{L}}^{ \pm, c_{L}, h_{L}}\left(\widetilde{\mathcal{B}}_{L, \epsilon}\right)}{L^{d-1}}=-\mathscr{Q}_{b, \xi}(m, \theta)+\inf _{\substack{\left|m^{\prime}\right| \leqslant m_{\star} \\ \theta^{\prime} \in[0,1]}} \mathscr{2}_{b, \xi}\left(m^{\prime}, \theta^{\prime}\right),
$$

where $\mathscr{2}_{b, \xi}(m, \theta)$ is as in (2.7).

Proof. We again begin with the representation (3.10)-(3.11) for the choices $h_{L} L^{d} \sim b L^{d-1}$ and $c_{L} L^{d} \sim \xi L^{d-1}$. For $m \in\left[-m_{\star}, m_{\star}\right]$ the last probability in (3.11) can be expressed from Assumption A and so the only thing to be done is the extraction of the exponential rate of $A_{W_{L}}^{\theta, c_{L}}(m)$ to within errors of order $o\left(L^{d-1}\right)$. This will be achieved Lemma 3.2, but before doing that, let us express the leading order behavior of the quantity $\Xi\left(m, \theta ; c_{L}\right)$. Noting the expansion $\mathscr{S}(p)=p \log p-p+O\left(p^{2}\right)$ for $p \downarrow 0$ we easily convince ourselves that

$$
\begin{aligned}
\Xi\left(m, \theta ; c_{L}\right)= & -\theta c_{L}\left(\log \frac{2 \theta c_{L}}{1+m}-1\right) \\
& -(1-\theta) c_{L}\left(\log \frac{2(1-\theta) c_{L}}{1-m}-1\right)+O\left(c_{L}^{2}\right) \\
= & c_{L}-c_{L} \log c_{L}+c_{L} \Upsilon(m, \theta)+O\left(c_{L}^{2}\right),
\end{aligned}
$$

where $\Upsilon(m, \theta)$ is as in (2.4). (The quantity $O\left(c_{L}^{2}\right)$ is bounded by a constant times $c_{L}^{2}$ uniformly in $m$ satisfying $|m| \leqslant 1-\eta$ and (3.6).) Invoking Lemma 3.2 and the facts that $\left|W_{L}\right|-L^{d}=O\left(L^{d-1}\right)$ and $L c_{L}^{2} \rightarrow 0$ as $L \rightarrow \infty$ we now easily derive that

$$
A_{W_{L}}^{\theta, c_{L}}(m)=\exp \left\{r_{L}+L^{d-1} \xi \Upsilon(m, \theta)+o\left(L^{d-1}\right)\right\},
$$

where $r_{L}=-L\left|W_{L}\right| c_{L} \log \left(c_{L} / e\right)$ is a quantity independent of $m$ and $\theta$. 
Putting the above estimates together, we conclude that

$$
\widetilde{K}_{L}(m, \theta)=\exp \left\{r_{L}-L^{d-1} \mathscr{Q}_{b, \xi}(m, \theta)+o\left(L^{d-1}\right)\right\}
$$

where $o\left(L^{d-1}\right)$ is small - relative to $L^{d-1}-$ uniformly in $m \in\left[-m_{\star}, m_{\star}\right]$ and $\theta \in[0,1]$. It remains to use this expansion to produce the leading order asymptotics of $P_{W_{L}}^{ \pm, c_{L}, h_{L}}\left(\widetilde{\mathcal{B}}_{L, \epsilon}\right)$. Here we write the latter quantity as a ratio,

$$
P_{W_{L}}^{ \pm, c_{L}, h_{L}}\left(\widetilde{\mathcal{B}}_{L, \epsilon}\right)=\frac{\widetilde{K}_{L, \epsilon}(m, \theta)}{Y_{L}}
$$

where $\widetilde{K}_{L, \epsilon}(m, \theta)$ is the sum of $\widetilde{K}_{L}\left(m^{\prime}, \theta^{\prime}\right)$ over all relevant values of $\left(m^{\prime}, \theta^{\prime}\right)$ that can contribute to the event $\widetilde{\mathcal{B}}_{L, \epsilon}$, while, we remind the reader, $Y_{L}$ is the sum of $\widetilde{K}_{L}\left(m^{\prime}, \theta^{\prime}\right)$ over all relevant $\left(m^{\prime}, \theta^{\prime}\right)$ 's regardless of their worth.

It is intuitively clear that the $r_{L}$-factors in the numerator and denominator cancel out and one is left only with terms of order $L^{d-1}$, but to prove this we will have to invoke a (standard) compactness argument. We first note that for each $\delta>0$ and each $(m, \theta) \in\left[-m_{\star}, m_{\star}\right] \times[0,1]$, there exists an $\epsilon>0$ and an $L_{0}<\infty$ - both possibly depending on $m, \theta$ and $\delta$ - such that, for $L \geqslant L_{0}$,

$$
\left|\frac{1}{L^{d-1}} \log \left(\widetilde{K}_{L, \epsilon}(m, \theta) e^{-r_{L}}\right)+\mathscr{Q}_{b, \xi}(m, \theta)\right| \leqslant \delta .
$$

(Here we also used that $\mathscr{Q}_{b, \xi}(m, \theta)$ is continuous in both variables on $\left[-m_{\star}, m_{\star}\right] \times[0,1]$.) By compactness of $\left[-m_{\star}, m_{\star}\right] \times[0,1]$, there exists a finite set of $\left(m_{k}, \theta_{k}\right)$ 's such that the above $\epsilon$-neighborhoods - for which (3.21) holds with the same $\delta-$ cover the set $\left[-m_{\star}, m_{\star}\right] \times[0,1]$. In fact we cover the slightly larger set

$$
\mathcal{R}=\left[-m_{\star}-\epsilon^{\prime}, m_{\star}+\epsilon^{\prime}\right] \times[0,1],
$$

where $\epsilon^{\prime}>0$. By choosing the $\epsilon^{\prime}$ 's sufficiently small, we can also ensure that for one of the $k$ 's, the quantity $\mathscr{2}_{b, \xi}\left(m_{k}, \theta_{k}\right)$ is within $\delta$ of its absolute minimum. Since everything is finite, all estimate are uniform in $L \geqslant L_{0}$ on $\mathcal{R}$.

To estimate $Y_{L}$ we will split it into two parts, $Y_{L, 1}$ and $Y_{L, 2}$, according to whether the corresponding $\left(m^{\prime}, \theta^{\prime}\right)$ belongs to $\mathcal{R}$ or not. By (3.21) and the choice of the above cover of $\mathcal{R}$ we have that $\frac{1}{L^{d-1}} \log Y_{L, 1}$ is within, say, $3 \delta$ of the minimum of $(m, \theta) \mapsto \mathscr{Q}_{b, \xi}(m, \theta)$ once $L$ is sufficiently large. (Here the additional $\delta$ is used to control the number of terms in the cover 
of $\mathcal{R}$.) On the other hand, Lemma 3.3 implies that $Y_{L, 2}$ is exponentially small relative to $Y_{L, 1}$. Hence we get

$$
\limsup _{L \rightarrow \infty}\left|\frac{1}{L^{d-1}} \log \left(Y_{L} e^{-r_{L}}\right)+\inf _{\substack{\left|m^{\prime}\right| \leqslant m_{\star} \\ \theta^{\prime} \in[0,1]}} \mathscr{Q}_{b, \xi}\left(m^{\prime}, \theta^{\prime}\right)\right| \leqslant 3 \delta
$$

Plugging these into (3.20) the claim follows by letting $\delta \downarrow 0$.

Proof of Theorem 2.1. This is a simple consequence of the compactness argument invoked in the last portion of the previous proof.

\subsection{Proof of Theorem 2.2}

Here we will prove Theorem 2.2 which describes the phase diagram for the "liquid" boundary condition, see the plot on the left of Fig. 1.

Proof of part (1). Our goal is to study the properties of the function $m \mapsto Q_{b, \xi}^{+}(m)$. Throughout the proof we will keep $J$ fixed (and larger than $J_{\mathrm{c}}$ ) and write $\mathscr{M}(\cdot)$ instead of $\mathscr{M}_{+, J}(\cdot)$. For $m \in\left[-m_{\star}, m_{\star}\right]$, let us define the quantity

$$
E_{\xi}(m)=-\xi g(m)+\mathscr{M}(m) .
$$

Clearly, this is just $Q_{b, \xi}^{+}(m)$ without the $b$-dependent part, i.e., $Q_{b, \xi}^{+}(m)=$ $-b m+E_{\xi}(m)$. Important for this proof will be the "zero-tilt" version of this function,

$$
\widehat{E}_{\xi}(m)=E_{\xi}(m)-E_{\xi}\left(-m_{\star}\right)-\left(m+m_{\star}\right) D_{E_{\xi}}^{\star},
$$

where $D_{E_{\xi}}^{\star}$ is the "slope of $E_{\xi}$ between $-m_{\star}$ and $m_{\star}$ ", see (2.11). Clearly, $E_{\xi}$ and $\widehat{E}_{\xi}$ have the same convexity/concavity properties but $\widehat{E}_{\xi}$ always satisfies $\widehat{E}_{\xi}\left(-m_{\star}\right)=\widehat{E}_{\xi}\left(m_{\star}\right)=0$.

Geometrically, the minimization of $Q_{b, \xi}^{+}(m)$ may now be viewed as follows: Consider the set of points $\left\{(m, y): y=E_{\xi}(m)\right\}$ - namely, the graph of $E_{\xi}(m)-$ and take the lowest vertical translate of the line $y=b m$ which contacts this set. Clearly, the minimum of $Q_{b, \xi}^{+}(m)$ is achieved at the value(s) of $m$ where this contact occurs. The same of course holds for the graph $y=\widehat{E}_{\xi}(m)$ provided we shift $b$ by $D_{E_{\xi}}^{\star}$. Now the derivative $\widehat{E}_{\xi}^{\prime}(m)$ is bounded below at $m=-m_{\star}$ and above at $m=m_{\star}$ (indeed, as $m \uparrow m_{\star}$ the derivative diverges to $-\infty)$. It follows that there exist two values, $-\infty<$ $b_{1}(\xi) \leqslant b_{2}(\xi)<\infty$, such that $m=m_{\star}$ is the unique minimizer for $b>$ 
$b_{1}(\xi), m=-m_{\star}$ is the unique minimizer for $b<b_{2}(\xi)$, and neither $m=m_{\star}$ nor $m=-m_{\star}$ is a minimizer when $b_{2}(\xi)<b<b_{1}(\xi)$.

On the basis of the above geometrical considerations, the region where $b_{1}$ and $b_{2}$ are the same is easily characterized:

$$
b_{1}(\xi)=b_{2}(\xi) \text { if and only if } \widehat{E}_{\xi}(m) \geqslant 0 \quad \forall m \in\left[-m_{\star}, m_{\star}\right]
$$

To express this condition in terms of $\xi$, let us define $T(m)=\mathscr{M}^{\prime \prime}(m) / g^{\prime \prime}(m)$ and note that $E_{\xi}^{\prime \prime}(m)>0$ if and only if $T(m)>\xi$. Now, for some constant $C=C(J)>0$,

$$
T(m)=C\left(m_{\star}-m\right)^{-\frac{d+1}{d}}(m+\cot (\kappa / 2))^{2},
$$

which implies that $T$ is strictly increasing on $\left[-m_{\star}, m_{\star}\right)$ with $T(m) \rightarrow$ $\infty$ as $m \uparrow m_{\star}$. It follows that either $\widehat{E}_{\xi}$ is concave throughout $\left[-m_{\star}, m_{\star}\right]$, or there exists a $T^{-1}(\xi) \in\left(-m_{\star}, m_{\star}\right)$ such that $\widehat{E}_{\xi}$ is strictly convex on $\left[-m_{\star}, T^{-1}(\xi)\right)$ and strictly concave on $\left(T^{-1}(\xi), m_{\star}\right]$. Therefore, by (3.26), $b_{1}(\xi)<b_{2}(\xi)$ if and only if $\widehat{E}_{\xi}^{\prime}\left(-m_{\star}\right)<0$, which is readily verified to be equivalent to $\xi>\xi_{\mathrm{t}}$. This proves part (1) of the theorem.

Proof of parts (3) and (4). The following properties, valid for $\xi>$ $\xi_{\mathrm{t}}$, are readily verified on the basis of the above convexity/concavity picture:

(a) For all $b_{2}(\xi)<b<b_{1}(\xi)$, there is a unique minimizer $m_{+}(b, \xi)$ of $m \mapsto Q_{b, \xi}^{+}(m)$ in $\left[-m_{\star}, m_{\star}\right]$. Moreover, $m_{+}(b, \xi)$ lies in $\left(-m_{\star}, T^{-1}(\xi)\right)$ and is strictly increasing in $b$.

(b) For $b=b_{1}(\xi)$, the function $m \mapsto Q_{b, \xi}^{+}(m)$ has exactly two minimizers, $m_{\star}$ and a value $m_{1}(\xi) \in\left(-m_{\star}, T^{-1}(\xi)\right)$.

(c) We have $b_{2}(\xi)=E_{\xi}^{\prime}\left(-m_{\star}\right)$.

(d) The non-trivial minimizer in (ii), $m_{1}(\xi)$, is the unique solution of

$$
E_{\xi}(m)+\left(m_{\star}-m\right) E_{\xi}^{\prime}(m)=E_{\xi}\left(m_{\star}\right) .
$$

Moreover, we have

$$
b_{1}(\xi)=E_{\xi}^{\prime}\left(m_{1}(\xi)\right) .
$$


(e) As $b$ tends to the boundaries of the interval $\left(b_{1}(\xi), b_{2}(\xi)\right)$, the unique minimizer in (a) has the following limits

$$
\lim _{b \downarrow b_{2}(\xi)} m_{+}(b, \xi)=-m_{\star} \quad \text { and } \quad \lim _{b \uparrow b_{1}(\xi)} m_{+}(b, \xi)=m_{1}(\xi)
$$

where $m_{1}(\xi)$ is as in (b). Both limits are uniform on compact subsets of $\left(\xi_{\mathrm{t}}, \infty\right)$.

Now, part (3) of the theorem follows from (a) while the explicit formula (2.13) for $b_{2}(\xi)$ for $\xi \geqslant \xi_{\mathrm{t}}$ is readily derived from (c). For, $\xi \leqslant \xi_{\mathrm{t}}$, the critical curve $\xi \mapsto b_{2}(\xi)$ is given by the relation $Q_{b, \xi}^{+}\left(m_{\star}\right)=Q_{b, \xi}^{+}\left(-m_{\star}\right)$, which gives also the $\xi \leqslant \xi_{\text {t }}$ part of (2.13). Continuity of $b \mapsto m_{+}(b, \xi)$ along the portion of $b=b_{2}(\xi)$ for $\xi>\xi_{\mathrm{t}}$ is implied by (e), while the jump discontinuity at $b=b_{1}(\xi)$ is a consequence of (a) and (e). This proves part (4) of the theorem.

Proof of part (2). It remains to prove the continuity of $b_{1}^{\prime}(\xi)$, identify the asymptotic of $b_{1}^{\prime}$ as $\xi \rightarrow \infty$ and establish the strict concavity of $\xi \mapsto b_{1}(\xi)$. First we will show that the non-trivial minimizer, $m_{1}(\xi)$, is strictly increasing with $\xi$. Indeed, we write (3.28) as $F_{\xi}(m)=0$, where $F_{\xi}(m)=E_{\xi}\left(m_{\star}\right)-E_{\xi}(m)-\left(m_{\star}-m\right) E_{\xi}^{\prime}(m)$. Now,

$$
\frac{\partial}{\partial \xi} F_{\xi}(m)=g(m)-g\left(m_{\star}\right)+\left(m_{\star}-m\right) g^{\prime}(m),
$$

which is positive for all $m \in\left[-m_{\star}, m_{\star}\right)$ by strict concavity of $g$. Similarly,

$$
\frac{\partial}{\partial m} F_{\xi}(m)=-E_{\xi}^{\prime \prime}(m)\left(m_{\star}-m\right)
$$

which at $m=m_{1}(\xi)$ is negative because $m_{1}$ lies in the convexity interval of $E_{\xi}$, i.e., $m_{1}(\xi) \in\left(-m_{\star}, T^{-1}(\xi)\right)$. From (d) and implicit differentiation we obtain that $m_{1}^{\prime}(\xi)>0$ for $\xi>\xi_{\text {t }}$. By (3.29) we then have

$$
b_{1}^{\prime}(\xi)=-\frac{g\left(m_{\star}\right)-g\left(m_{1}\right)}{m_{\star}-m_{1}}
$$

which, invoking the strict concavity of $g$ and the strict monotonicity of $m_{1}$, implies that $b_{1}^{\prime}(\xi)>0$, i.e., $b_{1}$ is strictly convex on $\left(\xi_{\mathrm{t}}, \infty\right)$.

To show the remaining items of (2), it suffices to establish the limits

$$
\lim _{\xi \downarrow \xi_{\mathrm{t}}} m_{1}(\xi)=-m_{\star} \quad \text { and } \quad \lim _{\xi \rightarrow \infty} m_{1}(\xi)=m_{\star} .
$$


Indeed, using the former limit in (3.33) we get that $b_{1}^{\prime}(\xi) \rightarrow-g^{\prime}\left(m_{\star}\right)$ as $\xi \rightarrow \infty$ while the latter limit and (c) above yield that $b_{1}^{\prime}(\xi) \rightarrow b_{2}^{\prime}\left(\xi_{\mathrm{t}}\right)$ as $\xi \downarrow \xi_{\text {t }}$ which in light of the fact that $b_{1}(\xi)=b_{2}(\xi)$ for $\xi \leqslant \xi_{\text {t }}$ implies the continuity of $b_{1}^{\prime}$. To prove the left limit in (3.34), we just note that, by (3.28), the slope of $\widehat{E}_{\xi}$ at $m=m_{1}(\xi)$ converges to zero as $\xi \downarrow \xi_{\text {t }}$. Invoking the convexity/concavity picture, there are two points on the graph of $m \mapsto$ $\widehat{E}_{\xi_{\mathrm{t}}}(m)$ where the slope is zero: $m_{\star}$ and the absolute maximum of $\widehat{E}_{\xi}$. The latter choice will never yield a minimizer of $Q_{b, \xi}^{+}$and so we must have $m_{1}(\xi) \rightarrow m_{\star}$ as claimed. The right limit in (3.34) follows from the positivity of the quantity in (3.31). Indeed, for each $m \in\left[-m_{\star}, m_{\star}\right)$ we have $F_{\xi}(m)>0$ once $\xi$ is sufficiently large. Hence, $m_{1}(\xi)$ must converge to the endpoint $m_{\star}$ as $\xi \rightarrow \infty$.

\subsection{Remaining Proofs}

Here we will prove Theorem 2.3, which describes the phase diagrams for the "ice" boundary condition, and Theorem 2.4 which characterizes the spin-sector of the distributions $P_{W_{L}}^{ \pm, c_{L}, h_{L}}$.

For the duration of the proof of Theorem 2.3, we will use the functions $E_{\xi}$ and $\widehat{E}_{\xi}$ from (3.24) to (3.25) with $\mathscr{M}=\mathscr{M}_{+, J}$ replaced by $\mathscr{M}=$ $\mathscr{M}_{-, J}$. The main difference caused by this change is that the function $m \mapsto$ $\widehat{E}_{\xi}(m)$ may now have more complicated convexity properties. Some level of control is nevertheless possible:

Lemma 3.5. There are at most two points inside $\left[-m_{\star}, m_{\star}\right]$ where the second derivative of function $m \mapsto \widehat{E}_{\xi}(m)$ changes its sign.

Proof. Consider again the function $T(m)=\mathscr{M}^{\prime \prime}(m) / g^{\prime \prime}(m)$ which characterizes $\widehat{E}_{\xi}^{\prime \prime}(m)>0$ by $T(m)>\xi$. In the present cases, this function is given by the expression

$$
T(m)=\frac{\mathscr{M}^{\prime \prime}(m)}{g^{\prime \prime}(m)}=C\left(m_{\star}+m\right)^{-\frac{d+1}{d}}(m+\cot (\kappa / 2))^{2}
$$

where $C=C(J)>0$ is a constant. Clearly, $T$ starts off at plus infinity at $m=-m_{\star}$ and decreases for a while; the difference compared to the situation in Theorem 2.2 is that $T$ now need not be monotone. Notwithstanding, taking the obvious extension of $T$ to all $m \geqslant-m_{\star}$, there exists a value $m_{\mathrm{T}} \in\left(-m_{\star}, \infty\right)$ such that $T$ is decreasing for $m<m_{\mathrm{T}}$ while it is increasing for all $m>m_{\mathrm{T}}$. Now two possibilities have to be distinguished depending on whether $m_{\mathrm{T}}$ falls in or out of the interval $\left[-m_{\star}, m_{\star}\right.$ ): 
(1) $m_{\mathrm{T}} \geqslant m_{\star}$, in which case the equation $T(m)=\xi$ has at most one solution for every $\xi$ and $m \mapsto \widehat{E}_{\xi}(m)$ is strictly concave on $\left[-m_{\star}, T^{-1}(\xi)\right.$ ) and strictly convex on $\left(T^{-1}(\xi), m_{\star}\right]$. (The latter interval may be empty.)

(2) $m_{\mathrm{T}}<m_{\star}$, in which case the equation $T(m)=\xi$ has two solutions for $\xi \in\left(T\left(m_{\mathrm{T}}\right), T\left(m_{\star}\right)\right]$. Then $m \mapsto \widehat{E}_{\xi}(m)$ is strictly convex between these two solutions and concave otherwise. The values of $\xi$ for which there is at most one solution to $T(m)=\xi$ inside $\left[-m_{\star}, m_{\star}\right]$ reduce to the cases in (1). (This includes $\xi=T\left(m_{\mathrm{T}}\right)$.)

We conclude that the type of convexity of $m \mapsto \widehat{E}_{\xi}(m)$ changes at most twice inside the interval $\left[-m_{\star}, m_{\star}\right]$, as we were to prove.

The proof will be based on studying a few cases depending on the order of the control parameters $\xi_{1}$ and $\xi_{2}$ from (2.18). The significance of these numbers for the problem at hand will become clear in the following lemma:

Lemma 3.6. The derivatives $\widehat{E}_{\xi}^{\prime}\left(m_{\star}\right)$ and $\widehat{E}_{\xi}^{\prime \prime}\left(m_{\star}\right)$ are strictly increasing functions of $\xi$. In particular, for $\xi_{1}$ and $\xi_{2}$ as defined in (2.18), we have

(1) $\widehat{E}_{\xi}^{\prime}\left(m_{\star}\right)<0$ if $\xi<\xi_{1}$ and $\widehat{E}_{\xi}^{\prime}\left(m_{\star}\right)>0$ if $\xi>\xi_{1}$.

(2) $\widehat{E}_{\xi}^{\prime \prime}\left(m_{\star}\right)<0$ if $\xi<\xi_{2}$ and $\widehat{E}_{\xi}^{\prime \prime}\left(m_{\star}\right)>0$ if $\xi>\xi_{2}$.

Proof. This follows by a straightforward calculation.

Now we are ready to prove the properties of the phase diagram for minus boundary conditions:

Proof of Theorem 2.3. Throughout the proof, we will regard the graph of the function $m \mapsto \widehat{E}_{\xi}(m)$ as evolving dynamically - the role of the "time" in this evolution will be taken by $\xi$. We begin by noting that, in light of the strict concavity of function $g$ from $(2.10)$, the value $\widehat{E}_{\xi}(m)$ is strictly decreasing in $\xi$ for all $m \in\left(-m_{\star}, m_{\star}\right)$. This allows us to define

$$
\tilde{\xi}_{\mathrm{t}}=\inf \left\{\xi \geqslant 0: \widehat{E}_{\xi}(m)<0 \text { for some } m \in\left(-m_{\star}, m_{\star}\right)\right\} .
$$

Now for $\xi=0$ we have $\widehat{E}_{\xi}(m)>0$ for all $m \in\left(-m_{\star}, m_{\star}\right)$ while for $\xi>\xi_{1}$, the minimum of $\widehat{E}_{\xi}$ over $\left(-m_{\star}, m_{\star}\right)$ will be strictly negative. Hence, we have $0<\xi_{\mathrm{t}} \leqslant \xi_{1}$.

We will also adhere to the geometric interpretation of finding the mimizers of $m \mapsto Q_{b, \xi}^{-}(m)$, cf. proof of part (1) of Theorem 2.2. In particular, for each $\xi>0$ we have two values $\tilde{b}_{1}$ and $\tilde{b}_{2}$ with $\tilde{b}_{2} \leqslant \tilde{b}_{1}$ such that 
the extremes $-m_{\star}$ and $m_{\star}$ are the unique minimizers for $b<\tilde{b}_{2}$ and $b>\tilde{b}_{1}$, respectively, while none of these two are minimizers when $\tilde{b}_{2}<b<\tilde{b}_{1}$. Here we recall that $\tilde{b}_{1}$ is the minimal slope such that a straight line with this slope touches the graph of $\widehat{E}_{\xi}$ at $m_{\star}$ and at some other point, but it never gets above it, and similarly $\tilde{b}_{2}$ is the maximal slope of a line that touches the graph of $\widehat{E}_{\xi}$ at $-m_{\star}$ and at some other point, but never gets above it.

As a consequence of the above definitions, we may already conclude that (1) is true. (Indeed, for $\xi \leqslant \tilde{\xi}_{\text {t }}$ we have $\widehat{E}_{\xi}(m) \geqslant 0$ and so the two slopes $\tilde{b}_{1}$ and $\tilde{b}_{2}$ must be the same. For $\xi>\tilde{\xi}_{\text {t }}$ there will be an $m$ for which $\widehat{E}_{\xi}(m)<0$ and so $\tilde{b}_{1} \neq \tilde{b}_{2}$.) The rest of the proof proceeds by considering two cases depending on the order of $\xi_{1}$ and $\xi_{2}$. We begin with the easier of the two, $\xi_{1} \geqslant \xi_{2}$ :

Case $\xi_{1} \geqslant \xi_{2}$ : Here we claim that the situation is as in Theorem 2.2 and, in particular, $\tilde{\xi}_{\mathrm{t}}=\xi_{1}$. Indeed, consider a $\xi>\xi_{2}$ and note that $\widehat{E}_{\xi}^{\prime \prime}\left(m_{\star}\right)>$ 0 by Lemma 3.6. Since $\widehat{E}_{\xi}^{\prime \prime}(m)$ is negative near $m=-m_{\star}$ and positive near $m=m_{\star}$, it changes its sign an odd number of times. In light of Lemma 3.5 , only one such change will occur and so $\left[-m_{\star}, m_{\star}\right]$ splits into an interval of strict concavity and strict convexity of $m \mapsto \widehat{E}_{\xi}(m)$. Now, if $\tilde{\xi}_{\text {t }}$ is not equal $\xi_{1}$, we may choose $\xi$ between $\tilde{\xi}_{\text {t }}$ and $\xi_{1}$ so that $\widehat{E}_{\xi}^{\prime}\left(m_{\star}\right)<$ 0 . This implies that $\widehat{E}_{\xi}(m)>0$ for all $m<m_{\star}$ in the convexity region; in particular, at the dividing point between concave and convex behavior. But then a simple convexity argument $\widehat{E}_{\xi}(m)>0$ throughout the concavity region (except at $-m_{\star}$ ). Thus $\widehat{E}_{\xi}(m)>0$ for all $m \in\left(-m_{\star}, m_{\star}\right)$ and so we have $\xi \leqslant \tilde{\xi}_{\mathrm{t}}$. It follows that $\tilde{\xi}_{\mathrm{t}}=\xi_{1}$.

Invoking the convexity/concavity picture from the proof of Theorem 2.2 quickly finishes the argument. Indeed, we immediately have (4) and, letting $\tilde{\xi}_{\mathrm{u}}=\tilde{\xi}_{\mathrm{t}}$, also the corresponding portion of (5). It remains to establish the properties of $\tilde{b}_{1}$ and $\tilde{b}_{2}-$ this will finish both (2) and (3a). To this end we note that $\tilde{b}_{1}$ is determined by the slope of $E_{\xi}$ at $m_{\star}$, i.e., for $\xi \geqslant \tilde{\xi}_{\mathrm{t}}$,

$$
\tilde{b}_{1}(\xi)=E_{\xi}^{\prime}\left(m_{\star}\right) .
$$

This yields the second line in (2.19); the first line follows by taking the slope of $E_{\xi}$ between $-m_{\star}$ and $m_{\star}$. As for $\tilde{b}_{2}$, here we note that an analogue of the argument leading to (3.33) yields

$$
\tilde{b}_{2}^{\prime}(\xi)=-\frac{g\left(m_{1}\right)-g\left(-m_{\star}\right)}{m_{1}+m_{\star}}, \quad \xi \geqslant \tilde{\xi}_{\mathrm{t}},
$$

where $m_{1}=m_{1}(\xi)$ is the non-trivial minimizer at $b=\tilde{b}_{2}(\xi)$. In this case the argument analogous to (3.31)-(3.32) gives $m_{1}^{\prime}(\xi)<0$. The desired limiting 
values (and continuity) of $\tilde{b}_{2}^{\prime}$ follow by noting that $m_{1}(\xi) \rightarrow m_{\star}$ as $\xi \downarrow \tilde{\xi}_{\mathrm{t}}$ and $m_{1}(\xi) \rightarrow-m_{\star}$ as $\xi \rightarrow \infty$.

Case $\xi_{1}<\xi_{2}$ : Our first item of business is to show that $\tilde{\xi}_{\mathrm{t}}<\xi_{1}$. Consider the situation when $\xi=\xi_{1}$ and $m=m_{\star}$. By Lemma 3.6 and continuity, the derivative $\widehat{E}_{\xi_{1}}^{\prime}\left(m_{\star}\right)$ vanishes, but, since we are assuming $\xi_{1}<\xi_{2}$, the second derivative $E_{\xi_{1}}^{\prime \prime}\left(m_{\star}\right)$ has not "yet" vanished, so it is still negative. The upshot is that $m_{\star}$ is a local maximum for $m \mapsto \widehat{E}_{\xi_{1}}(m)$. In particular, looking at $m$ slightly less than $m_{\star}$, we must encounter negative values of $\widehat{E}_{\xi_{1}}$ and, eventually, a minimum of $\widehat{E}_{\xi_{1}}$ in $\left(-m_{\star}, m_{\star}\right)$. This implies that $\tilde{\xi}_{\mathrm{t}}<\xi_{1}$.

Having shown that $\tilde{\xi}_{\mathrm{t}}<\xi_{1}<\xi_{2}$, we note that for $\xi \in\left(\tilde{\xi}_{\mathrm{t}}, \xi_{2}\right)$, the function $m \mapsto \widehat{E}_{\xi}(m)$ changes from concave to convex to concave as $m$ increases from $-m_{\star}$ to $m_{\star}$, while for $\xi \geqslant \xi_{2}$, exactly one change of convexity type occurs. Indeed, $\widehat{E}_{\xi}$ is always concave near $-m_{\star}$ and, when $\xi<\xi_{2}$, it is also concave at $m_{\star}$. Now, since $\xi>\tilde{\xi}_{t}$, its minimum occurs somewhere in $\left(-m_{\star}, m_{\star}\right)$. This implies an interval of convexity. But, by Lemma 3.5, the convexity type can change only at most twice and so this is all that we can have. For the cases $\xi>\xi_{2}$ we just need to realize that $\widehat{E}_{\xi}$ is now convex near $m=m_{\star}$ and so only one change of convexity type can occur. A continuity argument shows that the borderline situation, $\xi=\xi_{2}$, is just like $\xi>\xi_{2}$.

The above shows that the cases $\xi \geqslant \xi_{2}$ are exactly as for $\xi_{1} \geqslant \xi_{2}$ (or, for that matter, Theorem 2.2) while $\xi<\tilde{\xi}_{\text {t }}$ is uninteresting by definition, so we can focus on $\xi \in\left[\tilde{\xi}_{\mathrm{t}}, \xi_{2}\right.$ ). Suppose first that $\xi>\tilde{\xi}_{\mathrm{t}}$ and let $I_{\xi}$ denote the interval of strict convexity of $\widehat{E}_{\xi}$. The geometrical minimization argument then shows that, at $b=\tilde{b}_{1}$, there will be exactly two minimizers, $m_{\star}$ and a value $m_{1}(\xi) \in I_{\xi}$, while at $b=\tilde{b}_{2}$, there will also be two minimizers, $-m_{\star}$ and a value $m_{2}(\xi) \in I_{\xi}$. For $\tilde{b}_{1}<b<\tilde{b}_{2}$, there will be a unique minimizer $m_{-}(b, \xi)$ which varies between $m_{2}(\xi)$ and $m_{1}(\xi)$. Since $\widehat{E}_{\xi}$ is strictly convex in $I_{\xi}$, the map $b \mapsto m_{-}(b, \xi)$ is strictly increasing with limits $m_{1}(\xi)$ as $b \uparrow \tilde{b}_{1}(\xi)$ and $m_{2}(\xi)$ as $b \downarrow \tilde{b}_{2}(\xi)$. Both $m_{1}$ and $m_{2}$ are inside $\left(-m_{\star}, m_{\star}\right)$ so $m_{-}$undergoes a jump at both $\tilde{b}_{1}$ and $\tilde{b}_{2}$. Clearly, $m_{1}(\xi) \neq m_{2}(\xi)$ for all $\xi \in\left(\tilde{\xi}_{\mathrm{t}}, \xi_{2}\right)$.

At $\xi=\tilde{\xi}_{\text {t }}$, there will be an "intermediate" minimizer, but now there is only one. Indeed, the limits of $m_{1}(\xi)$ and $m_{2}(\xi)$ as $\xi \downarrow \tilde{\xi}_{t}$ must be the same because otherwise, by the fact that $\left[m_{1}(\xi), m_{2}(\xi)\right]$ is a subinterval of the convexity interval $I_{\xi}$, the function $\widehat{E}_{\tilde{\xi}_{t}}$ would vanish in a whole interval of $m$ 's, which is impossible. Denoting the common limit by $m_{0}$ we thus have three minimizers at $\xi=\tilde{\xi}_{\mathrm{t}}$; namely, $\pm m_{\star}$ and $m_{0}$. This proves part (4) and, letting $\tilde{\xi}_{\mathrm{u}}=\xi_{2}$, also part (5) of the theorem. As for the remaining parts, the strict concavity of $\tilde{b}_{1}$ and the limits (2.20) are again consequences of formulas of the type (3.33) and (3.37-3.38) and of the 
monotonicity properties of $m_{1}$ and $m_{2}$. The details are as for the previous cases, so we will omit them.

Proof of Theorem 2.4. As in Part I, the representation (2.28) is a simple consequence of the absence of salt-salt interaction as formulated in Lemma 3.1. The fact that any subsequential (weak) limit $\rho^{ \pm}$of $\rho_{L}^{ \pm}$has all of its mass concentrated on the minimizers of $Q_{b, \xi}^{ \pm}$is a consequence of Theorem 2.1 and the fact that $m$ can only take $O(L)$ number of distinct values. Moreover, if the minimizer is unique, which for the plus boundary conditions happens when $b \neq b_{1}(\xi), b_{2}(\xi)$, any subsequential limit is the Dirac mass at the unique minimum (which is $m_{+}(b, \xi)$ for the plus boundary conditions and $m_{-}(b, \xi)$ for the minus boundary conditions).

\section{ACKNOWLEDGMENTS}

The research of K.S.A. was supported by the NSF under the grants DMS0103790 and DMS-0405915. The research of M.B. and L.C. was supported by the NSF grant DMS-0306167.

\section{REFERENCES}

1. K. Alexander, M. Biskup, and L. Chayes, Colligative properties of solutions: I. Fixed concentrations, previous paper.

2. K. Alexander, J.T. Chayes, and L. Chayes, The Wulff construction and asymptotics of the finite cluster distribution for two-dimensional Bernoulli percolation, Commun. Math. Phys. 131:1-51 (1990).

3. M. Biskup, L. Chayes, and R. Kotecký, On the formation/dissolution of equilibrium droplets, Europhys. Lett. 60:21-27 (2002).

4. M. Biskup, L. Chayes, and R. Kotecký, Critical region for droplet formation in the twodimensional Ising model, Commun. Math. Phys. 242:137-183 (2003).

5. T. Bodineau, The Wulff construction in three and more dimensions, Commun. Math. Phys. 207:197-229 (1999).

6. T. Bodineau, D. Ioffe, and Y. Velenik, Rigorous probabilistic analysis of equilibrium crystal shapes, J. Math. Phys. 41:1033-1098 (2000).

7. R. Cerf and A. Pisztora, On the Wulff crystal in the Ising model, Ann. Probab. 28:9471017 (2000).

8. R.L. Dobrushin, R. Kotecký, and S.B. Shlosman, Wulff Construction. A Global Shape from local interaction (Amer. Math. Soc., Providence, RI, 1992).

9. R.L. Dobrushin and S.B. Shlosman, in: Probability Contributions to Statistical Mechanics (Amer. Math. Soc., Providence, RI, 1994), pp. 91-219.

10. P.E. Greenwood and J. Sun, Equivalences of the large deviation principle for Gibbs measures and critical balance in the Ising model, J. Statist. Phys. 86:149-164 (1997).

11. P.E. Greenwood and J. Sun, On criticality for competing influences of boundary and external field in the Ising model, J. Statist. Phys. 92:35-45 (1998).

12. D. Ioffe and R.H. Schonmann, Dobrushin-Kotecký-Shlosman theorem up to the critical temperature, Commun. Math. Phys. 199:117-167 (1998). 
13. R. Kotecký and I. Medved', Finite-size scaling for the 2D Ising model with minus boundary conditions, J. Statist. Phys. 104:905-943 (2001).

14. J.L. Lebowitz and C.-E. Pfister, Surface tension and phase coexistence, Phys. Rev. Lett. 46:1031-1033 (1981).

15. T. Neuhaus and J.S. Hager, $2 d$ crystal shapes, droplet condensation and supercritical slowing down in simulations of first order phase transitions, J. Statist. Phys. 113:47-83 (2003).

16. R.H. Schonmann and S.B. Shlosman, Complete analyticity for 2D Ising completed, Commun. Math. Phys. 170:453-482 (1995).

17. R.H. Schonmann and S.B. Shlosman, Constrained variational problem with applications to the Ising model, J. Statist. Phys. 83:867-905 (1996). 